\title{
TMX-UPGRADE (IMX-U) OPERATION IN THE SLOSHING-ION MODE
}

T. C. Simonen, S. L. Allen, T. A. Casper, J. F. Clauser, F. H. Coensgen,

D. L. Corre]1, W. F. Cummins, C. C. Darm, J. H. Foote, R. K. Goodman, D. P. Grubb, E. B. Hooper, R. S. Hornady, A. L. Hunt, R. G. Kerm, A. W. Molvik, R. H. Munger, W. E. Nexsen, T. J. Orzechowski,

W. L. Pickles, P. Poulsen, M. E. Rensink, B. W. Stallard, and W. C. Turner Lawrence Liveralore National Laboratory, University of California

$$
\begin{gathered}
\text { W. Hsu and W. Bauer } \\
\text { Sandia Corp., Livermore }
\end{gathered}
$$

C. A. Clower, B. H. Failor and M. D. Flammer Department of Applied Science, U.C. Davis - Livermore Campus

T. L. Yu and R. Zimmermann Johns Hopk ins University

R. F. Ellis and C. J. Lasnier University of Maryland

September 24, 1982

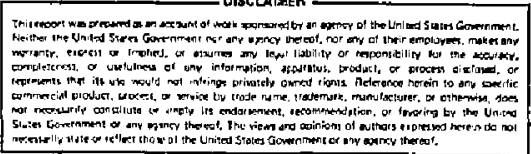




\section{INTRODUCTION}

This report sumarizes initial results from TMX-U carried out from June through August 1982. In these successful experiments we operated TMX-U in the sloshing-ion mode. We generated sloshing ions, measured improved energy confinement, and observed improved microstability compared to TMX. The experiments operated about as we expected and we are pleased with ti.e results. During this period many additional achievements were also recorded. The magnetically confined sloshing ions constitute one of the two ingredients needed to build a thermal barrier. The second ingredient consists of magnetically confined electrons, which will be studied in ihe next series of $T M X-U$ experiments using microwave heating of the electrons. Later, the hot ions and electrons will be combined to form thermal barriers.

\section{TMX-UPGRADE}

Figure 1 illustrates the key components of TMX-U. The operating characteristics of TMX-U during the experiments reported here are given in Table I. An extensive array of diagnostics was employed for these experiments, and 193 diagnostic signals were recorded on each shot. Many of these instruments (listed in Table II) were similar to those used on TMX. During this period, 637 data shots were taken (as shown in Fig. 2). We first operated with hydrogen (200 shots) but switched to deuterium which gave the better results described here. The improvement obtained with deuterium is attributed to three facts: First, cold deuterium gas penetrates less deeply into the plasina and thereby causes less chargeexchange loss. Second, the beam-trapping efficiency is $40 \%$ higher. Third, the neutral beams operated better on deuterium ard with less gas input. He could nevertheless obtain full-duration plasma shots with hydrogen as shown in Fig. 3.

Tandem mirrors $c$ an be operated in many ways. Figure 4 illustrates three operating modes. Shown schematically at top left is the magneticfield axial profile for one-half of a tandem mirror. Below we show the corresponding axial potential profiles which confine central-cell ions not 


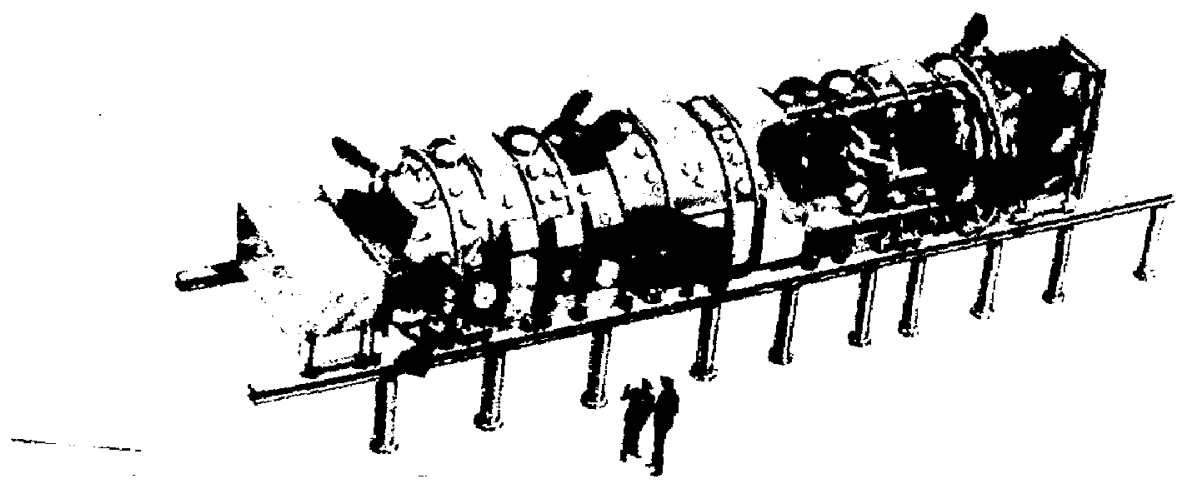

Fig. 7. Cutaway drawing of TMX. 
Table 1. Operating Parameters of TMX-U During Sloshing-Ion Experiments

Magnet:

$\begin{array}{lc}\text { End-plug midplane field, } T & 0.5 \\ \text { Plug-mirror ratio } & 4: 1 \\ \text { Plug length, m } & 3.0 \\ \text { Central-cel] length } & 8.0 \\ \text { Central-cell strength, } T & 0.3 \\ \text { Central-cell limiter radius, m } & 0.32\end{array}$

Neutral Beam:

Species

Duration, ms

$\mathrm{D}_{2}$

Total power, MW

75

Number of plug beams

Plug-injection angles,

6

Number of central-celt beams

$47^{\circ}$

Central-cel1 injection angles

6

$70^{\circ}, 58.5^{\circ}$

ECRH:

Number of gyrotrons 1

Maximum power to plasma, $\mathrm{kW} \quad 60$

Frequency, GHz 28

Antenna lacation, T 1.0 
Table I1. Number and Locations of Initial TMX U Diagnostics Instruments: August 1982.

\begin{tabular}{|c|c|c|c|c|c|c|c|c|}
\hline \multirow[t]{2}{*}{ Diagnostics } & \multicolumn{3}{|c|}{ East plug } & \multirow{2}{*}{$\begin{array}{c}\text { Central } \\
\text { cell }\end{array}$} & \multicolumn{3}{|c|}{ West plug } & \multirow[t]{2}{*}{ Total } \\
\hline & Wall & Plug & Barrier & & Barrier & Plug & WaIt & \\
\hline Thomson scattering & -- & -- & -- & 1 & -- & -- & -- & 1 \\
\hline Beam attenuation & -- & $\rightarrow$ & 22 & 5 & 22 & -- & -- & 49 \\
\hline Microwave interferometer & -- & $I$ & 1 & 1 & 1 & -- & -- & 4 \\
\hline Diamagnetic loop & -- & 2 & 1 & 8 & 1 & 2 & -- & 14 \\
\hline$x$ rays & -- & -- & 1 & -- & -- & - & $=-$ & 1 \\
\hline End-loss analyzer & 2 & -- & -- & - & -- & -- & 2 & 4 \\
\hline Faraday cups & 14 & -- & -- & -- & -- & -- & 14 & 28 \\
\hline Net current disks & 14 & - & -- & -- & -- & -- & 14 & 28 \\
\hline Ultraviolet & -- & 1 & -- & 1 & -- & $=$ & -- & 2 \\
\hline Secondary-emission detecturs & -- & -- & 21 & 4 & 7 & -- & $=-$ & 32 \\
\hline RF probes & -- & 8 & -- & 8 & -- & 5 & -- & 21 \\
\hline Gas pressure & 1 & - & 1 & 1 & 1 & $=$ & 1 & 5 \\
\hline Microwave emission & -- & -- & 1 & -- & -- & -- & -- & 1 \\
\hline Microwave scattering & -- & - & 1 & -- & -- & -- & -- & 1 \\
\hline Langmuir probe & -- & - & 1 & -- & $=-$ & -- & -- & 1 \\
\hline Surface probe & $=$ & $=$ & 1 & $=$ & $=$ & $=$ & \pm & 1 \\
\hline TOTAL & 31 & 12 & 51 & 29 & 32 & 7 & 31 & 193 \\
\hline
\end{tabular}




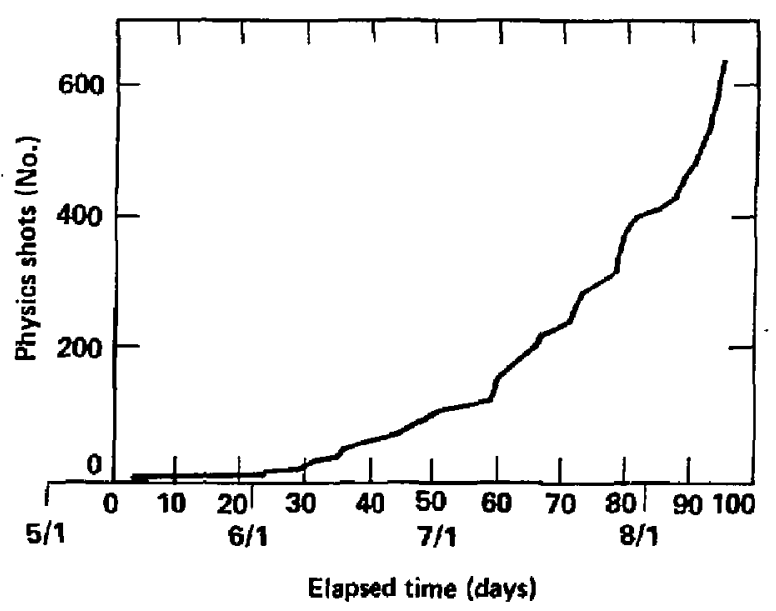

Fig. 2. Cumulative TMX-U data production. 

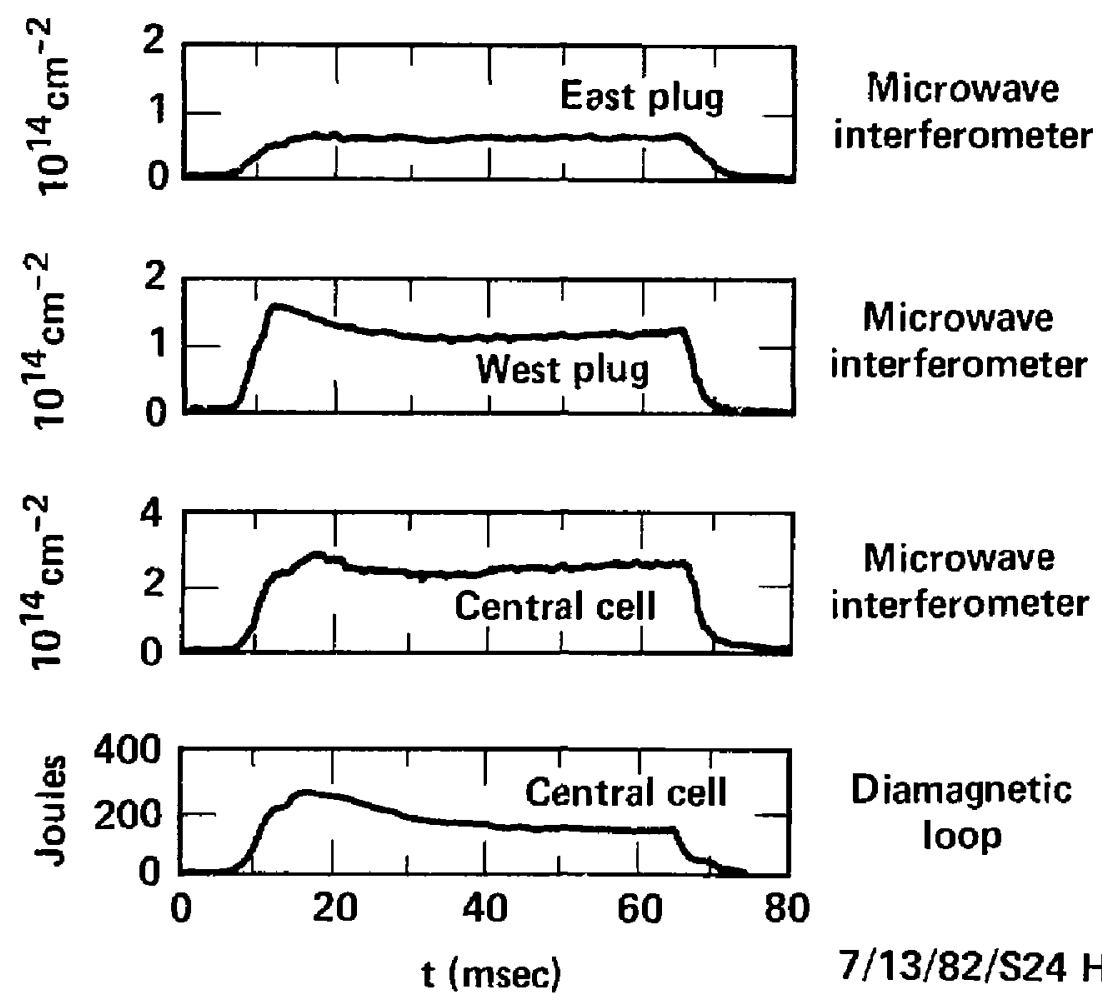

Diamagnetic loop $7 / 13 / 82 / \mathrm{S}_{24} \mathrm{H}_{2}$

Fig. 3. Example of 75-ms duration shot (operation with hydrogen). 


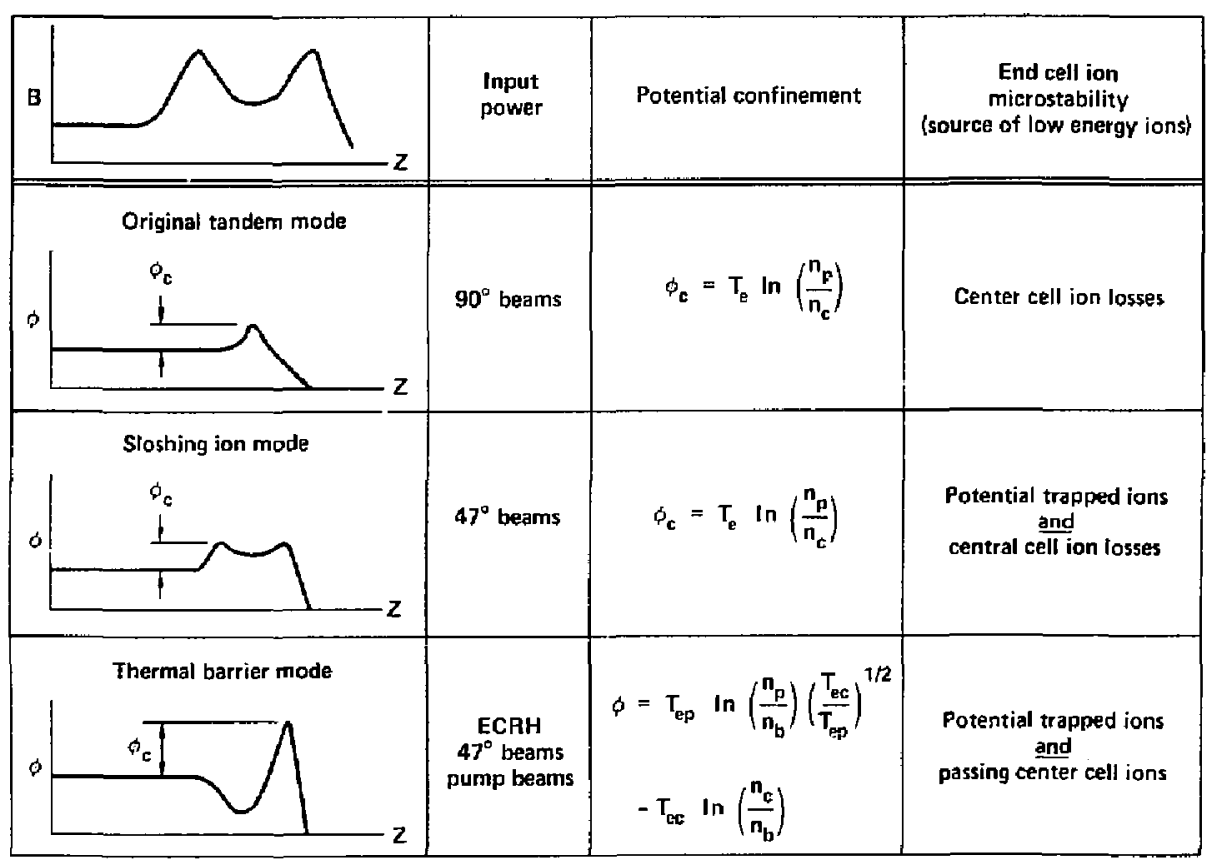

Fig. 4. Tandem-mirror operating modes. 
confined by the magnetic mirror. We indicate how each mode is generated, how the potential is determined, and the source of low-energy ions that maintains microstability of the beam-injected plug ions.

In the original tandem mode, end-cell beams were injected at $90^{\circ}$ to the magnetic field lines to generate a higher density in the end plug than in the central cell. The potential is determined by the Boltzman relation and microstability is maintained by central-cell-ion losses. This is the operating mode of our previous TMX experiment.

In the sloshing-ion mode, end-cell beams are injected at an angle to the field lines. In this case the ions slosh, or bounce back-and-iorth, generating density peaks at their turning points - away from the bottom of the mirror. Now the axial potential profile has a dip that can electrostatically confine low-energy ions to aid loss-cone stability. As in the original tanden mode, central-cell-ion losses provide additional stability. Beam injection at an angle also reduces the anisotropy drive of Alfuen-jon-c.yclotron mode, which was present in the previous TMX experiment. This is the initial operating mode of TMX-U and is described in the following section.

In thermal-barrier experiments the confining potential will be increased with ECRH by increasing the plug electron temperature above the central-cell electron temperature. In the thermal-barrier. mode, we expect further microstability to be achieved with potential-trapped ions and passing central-cell ions, without relying on central-cell josses for microstability.

\section{PRODUCTION OF STEADY-STATE PLASMAS}

\section{Vacuum Conditions}

Reducing the amount of neutral gas outside the plasma radius, characterizing the species present, and increasing the reliability of the vacuum system were among the goals undertaken for TMX-U. These goals have been accomplished with the installation of warm plasma liners and upgrading of the vacuum-system components, particularly the getter system and pressure- 
measuring diagnostic system. In TMX $U$, we have achieved higher reliability, shorter cleanup times for plasma operation, and better understanding of gas dynamics. We baked the machine to remove water and glaw discharged to remove hydrocarbons.

Figure 5 shows the vessel pressure during a typical day of operation. Base pressure of $3 \times 10^{-6}$ Torr during neutral-beam workup are common in the morning. Pressures near $1 \times 10^{-8}$ Torr $\left(\mathrm{CH}_{4}\right)$ are routine with the liquid nitrogen (LN) liners chilled and full operation on a 5- or 6-minute shot interval. When the shot sequence is completed, the LN supply to the liners is turned off, and methane $\left(\mathrm{CH}_{4}\right)$ begins to "wick" of $f$ the liners almost immediately. The methane dump continues for 5 or 6 hours and the vessel returns to $2 \times 10^{-6}$ Torr before 4:00 a.m.

The TMX-U getter system operates for one minute: initially, in the outer first-injector region, then in the second-injector region, and finally inside the plasma region defined by the warm plasma liner. The getter wires are kept warm at all times by a 20-A minimum current. This reduces wire failure because of current-turnon overshoot during each 1 -minute getter cycle, maintains a temperature of $100^{\circ} \mathrm{C}$ on the walls facing the plasma, and prevents pumping of gas by the getter wires between shots. All getter power is off for 30 seconds just before the plasma shot to allow methane pump-out and allow getter shutters to open. We feel these getter procedures account for the short one-day operation time required on TMX-U to produce full-duration (75 ms) plasmas. In spite of these improvements, since vacuum requirements are very severe in TMX-U, cold gas charge exchange is an important power-loss process as described later.

\section{ECRH Startup}

In these experiments, we demonstrated plasma startup using an ECRH pulse to ionize gas from the central-cell gas feed and from neutral beans. This technique is an attractive alternative to using plasma guns at the end walls. He show in fig. 6, several measurements made during a shot initiated by a 20-ms ECRH pulse and without stream guns. These measurements represent a few of the approximateiy 200 diagnostic signals recorded on each shot. Shown are the density, diamagnetism, charge-exchange flux, and end-loss 
$9 \times 10^{-9}$ (torr $\mathrm{CH}_{4}$ ) BASE PRESSURES ARE ROUTINE THROUGH A DAY OF PHYSICS SHOTS. $\mathrm{CH}_{4}$ DESORBTION FROM THE LINERS OCCURS AS THEY WARM UP

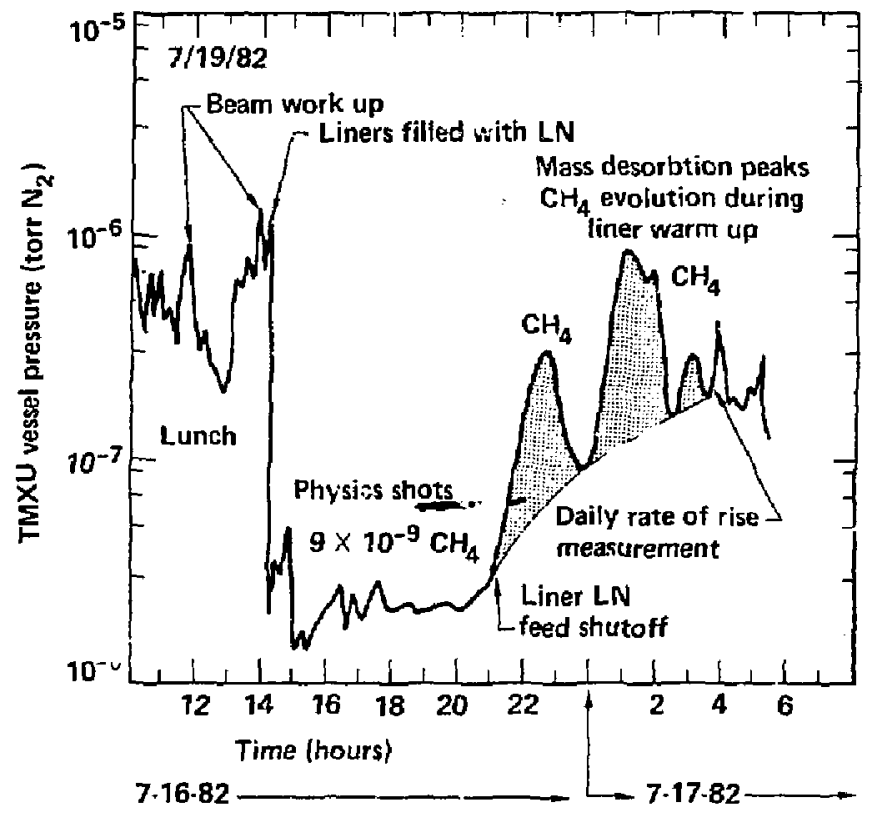

Fig. 5. Vacuum-pressure history during one day of operation. 

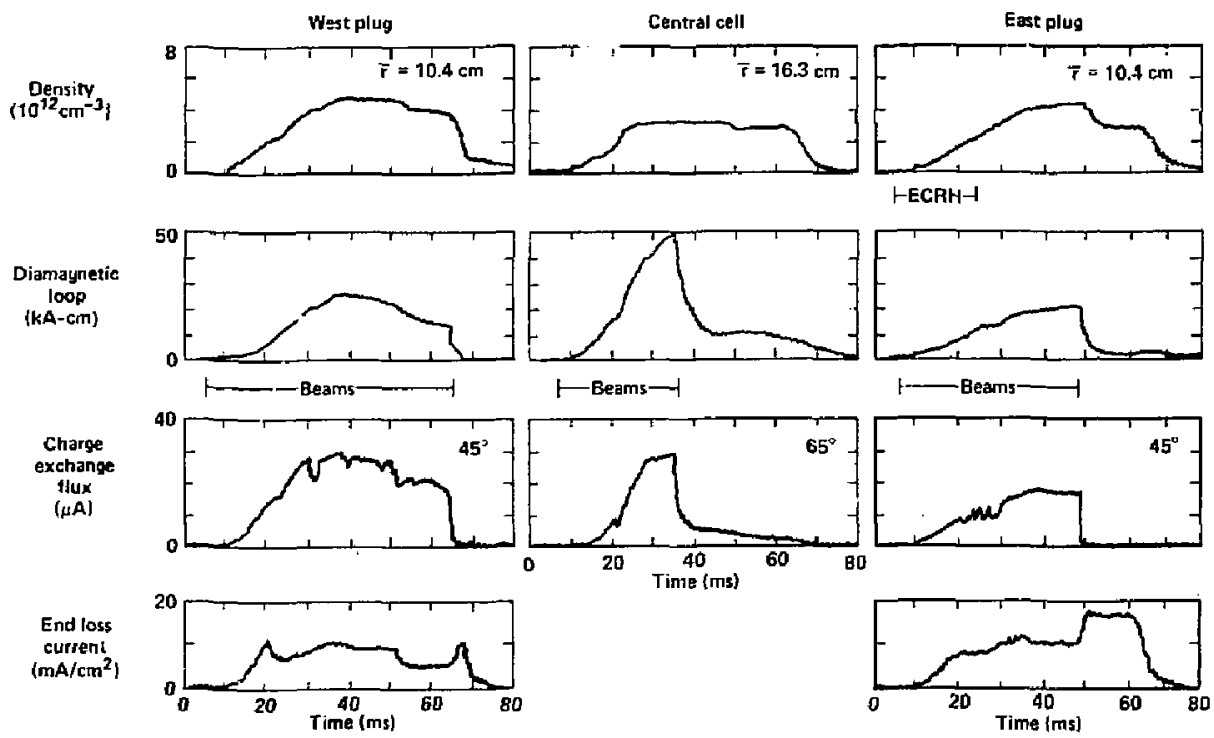

Fig. 6. Typical diagnostic signals with ECRH start up (operation with deuterium). 
current from both plugs and the central cell during the time interval from 0 to $80 \mathrm{~ms}$. Neutral beams are turned off at different times to characterize the plasma. Short neutral-beam interrupts introduce the variations present in the charge-exchange and diamagnetic signals.

In this shot, densities reach the mid $10^{12}$ range, the mean ior energy of the plugs is $5 \mathrm{keV}$, and the mean ion energy of the central cell is $2 \mathrm{keV}$ while the central-cell beams are on. We believe the plug ion distribution is composed of a 10-keV component and a comparable density of approximately $100-\mathrm{eV}$ ions.

The central-ce11 electron temperature reached $100 \mathrm{eV}$, about as expected. The low electron temperature in tinese experiments is a result of the high central-cell gas-feed currents needed to sustain the plasma. When we attempted to decrease the gas input, trie plasma was not sustained. We attribute this to a reduction in edge-plasma density and thereby an inability to shield the core plasma froril cold-gas charge-exchange loss. When the gas feed was reduced, no increase in fluctuations were observed, indicating that microinstabilities were not limiting the operation. In the thermal-barrier operating mode, $T_{e}$ is expected to be higher.

\section{MHD Stability}

MHD stability is evaluated from diamagnetic measurements in the central cell and end plugs. From these measurements and from measurements of the plasma radius, we determine the plasma beta, assuming a uniform radial temperature profile. We found that contral-cell betas up to $10 \%$ could be achieved, and we did not observe any limitations as the central-cell neutral-beam power was increased. Likewise, end-plug betas up to $4 \%$ were achieved. For comparison, in the thermal-barrier mode we are aiming for $20 \%$ central-ce 11 beta and $3 \%$ end-plug ion beta (together with $30 \%$ hot-electron end-piug beta).

As a test of MHD stability, we carried out experiments in which we turned off the end-plug neutral beams while continuing central-cell beam injection. He found that the end-plug beta decayed in a few milliseconds while the central cell decayed with a stower 5- to l0-ms time constant. During this smooth decay process, we were able to document central-cell betas up to 18 times larger than the end-plug betas (see Fig. 7), with no abrupt plasma terminations. 
- maXimum betas (NO mhD limitation OBSERVED)

ANCHOR $=B_{A}=4 \%$

CENTRAL CELL $=B_{C}=10 \%$

- $B_{c}$ UP T0 $78 \times B_{A}$

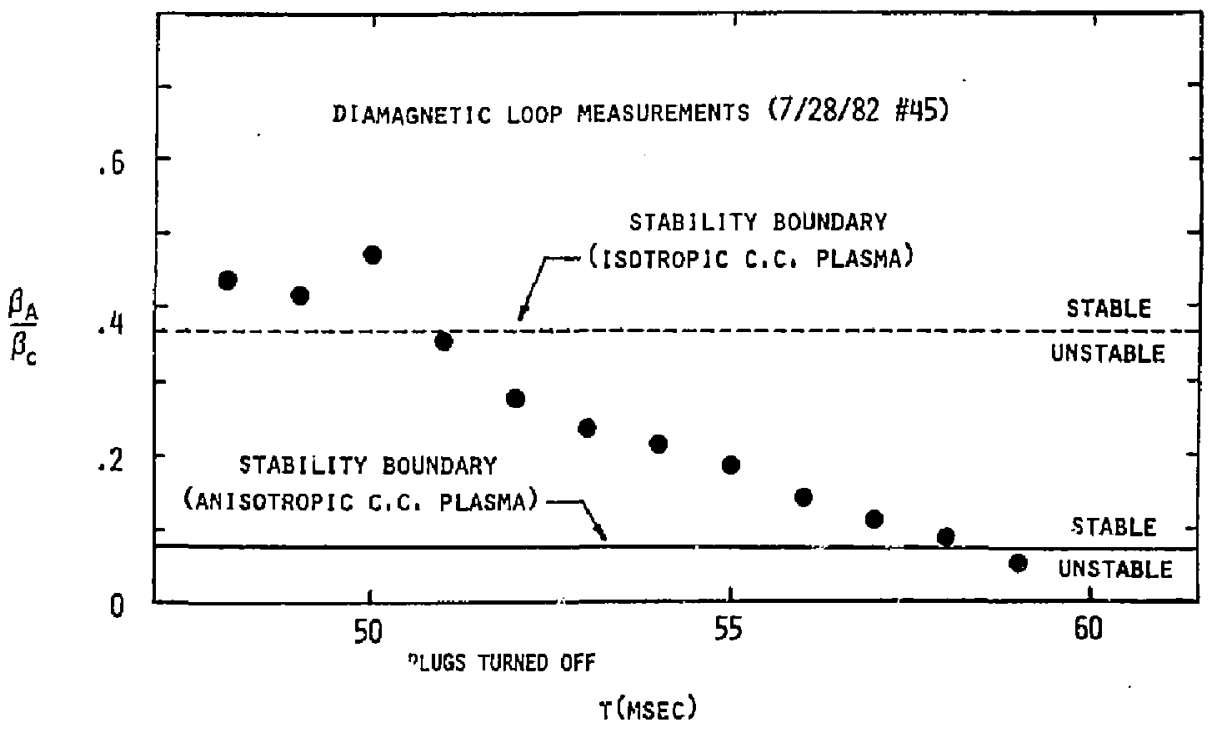

Fig. 7. End-f]ug betas as low as $5 \%$ of the central-cell beta provide MHD stabjity in TMX-U. 
4. Energy Confinement and Power Balance

The overall energy-confinement time is $\tau_{E}=$ stored plasma energy/trapped-beam power. The stored plasma energy is measured by the diamagnetic loops and the beam power input from the beam-attenuation diagnostic. Results of this caiculation for several TMX-U shots are shown in Fig. 8. Overall energy-confinement times appraaching $5 \mathrm{~ms}$ were produced, which is at least a three times longer time than in TMX. We attribute this improvement to better vacuum conditions (and thus lower charge-exchange losses) and to improved microstability.

We completed a preliminary assessment of a global power balance in the sloshing-ion mode without central-cell beams and find that axial particle loss accounts for at least $50 \%$ of the trapped-beam power. Charge-exchange loss of the pluy ions is believed to be an additional important loss process.

When we examine the power flow we find that, within experimental uncertainties of a factor of two, axial particle loss accounts for the power transferred to the electrons by Coulomb drag on the plug ions. Central-cell bulk in drag on the electrons, however, may not by the only power input to the central-cell ions. The central-cell bulk ion temperature is approximately the same as the electron temperature, indicating tiiere is a source of centrai-cell ion heating in addition to power transfer from the central-cell electrons.

\section{Electrostatic Plugging}

While operating in the sloshing-ion mode, we were able to abtain electrostatic plugging of the central-cell bulk ions when the plug density was greater than that of the central-cel1. The results of data analysis agree with theory and show that we were able to improve the central-cell confinement by a factor of two with plugging. Figure 6 shows that the eastend-Joss current increases when the east plug beams are turned off.

The maximum ratio between $p l u g$ and central-cell density in these experiments was $2: 1$. Higher plug densities were limited by the available neutral-beam current. Lower central-cell densities were limited by the 


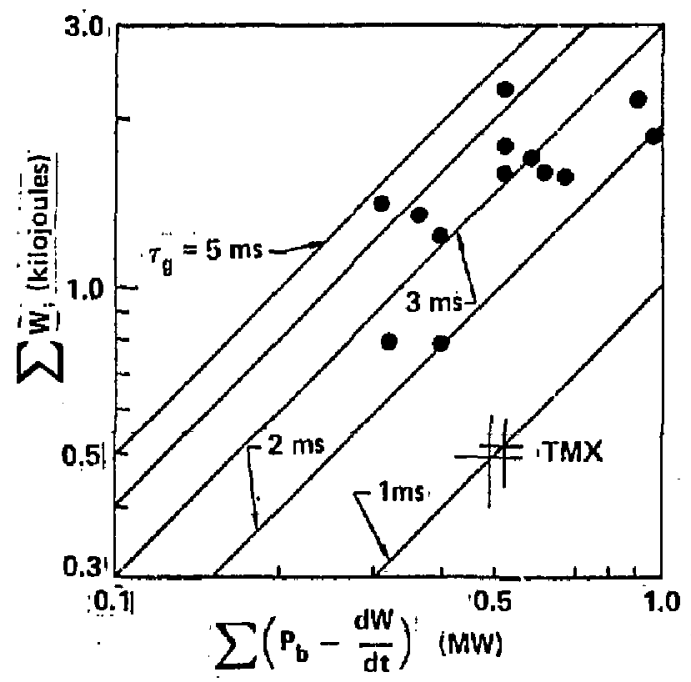

Fig. 8. Larger plasma-energy content of TMK-U, relative to TMX, for comparable input beam power indicates better global-energy confinement in TMX-U. 
inability to reduce central-cell fueling without loss of the plug density. This is because a decrease in the halo plasma density shields the hat plug ions from charge-exchange loss of $f$ the cold background gas.

Preliminary analysis of other data indicates that we were also able to obtain enhanced confinement with plugging when the plug and central-cell densities were approximateiy equal $\left(n_{p} / n_{c} v 1\right)$. This result is not explained by tandem-mirror theory without thermal barrier. It is, however, a potentially important finding, because it means we have already obtained evidence of plugging in a tandem mirror where the plug density is less than or equal to that of the central cell (which we hope to obtain in later therinal-barrier operation).

\section{Impurity Measurements}

Impurity concentrations were measured in the central cell with a 1024channel time-resolving EUV spectrograph and in the east-end plug with a 22channel radial EuN monochromator. A spectrum from the central cell is shown in Fig. 9. The impurity concentrations are $1 \%$ or less and are typically $0 \leq 0.5 \%, N \leq 0.3 \%$, and $C \leq 0.2 \%$. We conclude that TMX-U, similar to $T M X$, had low impurity concentrations.

\section{SLOSHING-ION MEASUREMENTS}

To establish the existence of sloshing ions, we used (1) an angular array of secondary-emission detectors (SFDs, which measure charge-exchange flux; (2) an axial array of diamagnetic loops, which measure perpendicular pressure; and ( 3 ) microwave interferometers at the midplane $(B=0.5 \mathrm{~T})$ and near the turning point $(B=0.8 \mathrm{~T})$, which measure electron density. Solid-. state and resistance-probe measurements substantiate that the SED array is measuring ions with energy exceeding $5 \mathrm{keV}$. Both the SEDs and diamagnetic loops always show the presence of sloshing ions; angular distribution peaked near $47^{\circ}$, and pressure peaked off the midplane. The microwave interferometer measures electron density associated with both hot sloshing ions and cold ions. It measures a density at the turning point equal to or up to twice the midplane density, thus providing mare evidence for the presence of sloshing ions. 


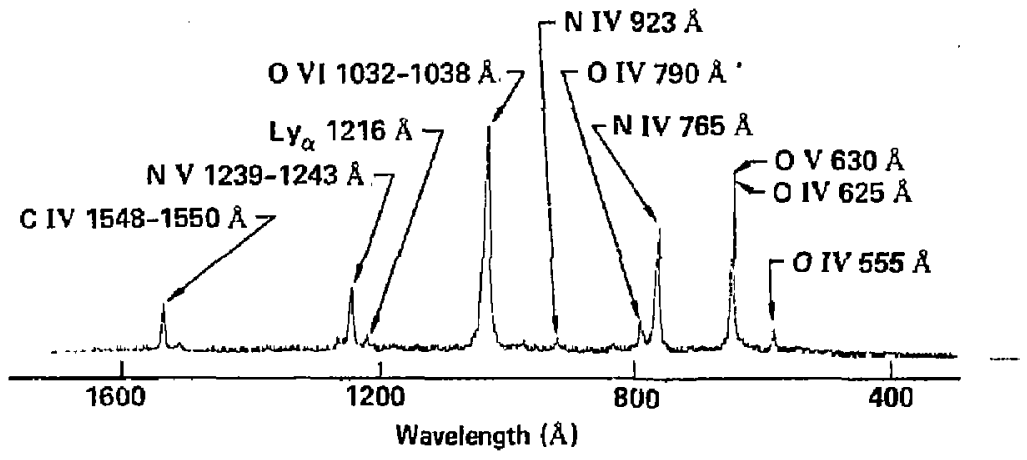

Fig. 9. Ultraviolet-emission spectrum from TMX-U indicates low impurity concentrations. 
Figure 10 shows measurements of sloshing ions and a comparison with a Fokker-Planck Code. The angular array of charge-exchange detectors shows that the sloshing-ion distribution remains peaked. Near the injection angle, agreement with the code is good. However, the measurements show more ions than predicted near $90^{\circ}$. In future experiments, measurements will be made to determine the energy and density of these ions.

An axial array of diamagnetic loops a]so substantiates the presence of sloshing ions. On the right is shown the axial magnetic-field profile for one-half of an end plug. The diamagnetic lonp data shows a higher perpendicular pressure near the sloshing-ion turning point, in agreement with the code.

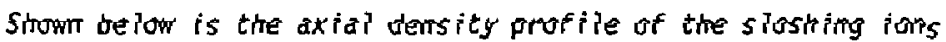
determined from the angular array of charge-exchange detectors. In addition to the sloshing ions, there is a comparable density of low-energy ions. Thus, TMX $U$ has achieved densities in the mid $10^{12} \mathrm{~cm}^{-3}$ range as required for the thermal-barrier operating mode. We conclude that by and large the sloshing ions are behaving as expected. We have definitely generated a hot-ion density located off the midplane, as required for the thermal-barrier mode.

\section{ION MICROSTABILITY}

To evaluate the microstability of the sloshing-ion distribution, we have used electrostatic probes ( end piog and in the central celi. He fird the slosing-ion mote to be more microstable than it was in TMX (which operated in the original tandem-mirror mode). In TMX-U we have not observed degradation of confinement because of fluctiations. The level of fluctuations has changed by a factor of three but plasma parameters remain unchanged.

With the exception of the sloshing-ion feature, TMX $U$ is actually more vulnerable to microinstability than was TMX (as outlined in Table III) because it has sharper raoial-density gradients and a longer plug length. However, in comparison to TMY, TMX-U fluctuation amplitudes are smaller (less than $1 V$ when measured at a radius equal to twice the plasma radius). These fluctuations exhibit characteristics of a loss-cone driven mode. The frequency is that of the ion-cyclotron frequency outside the sloshing-ion density peak where warm ions would not be electrostatically confined. 

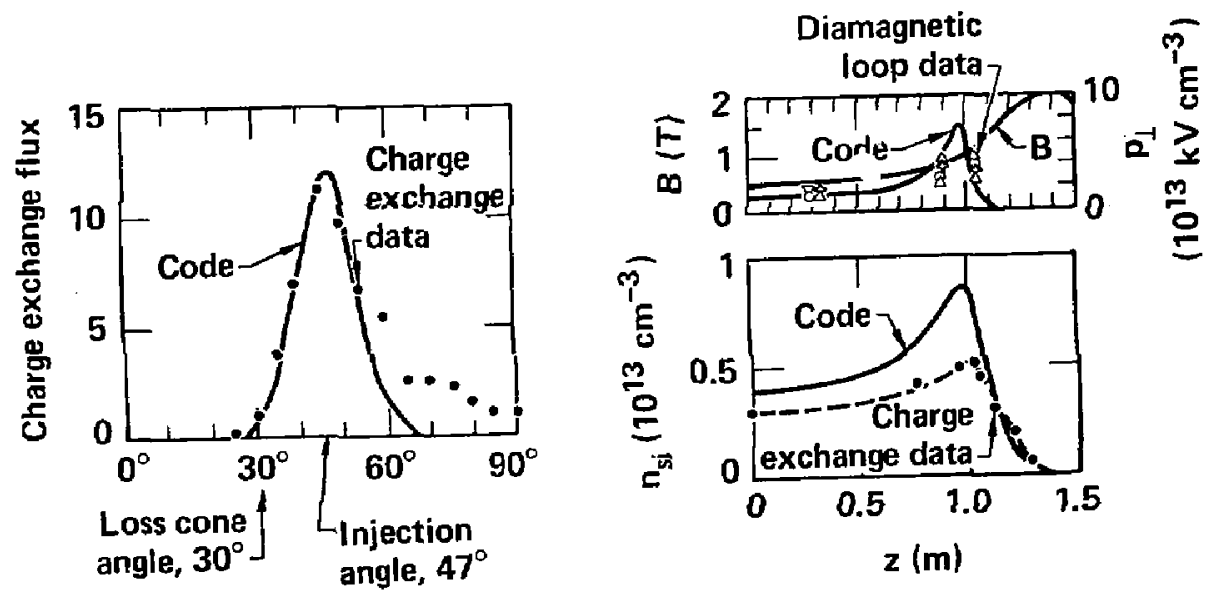

Fig. 10. Measurements of sloshing ions: angular distribution (left), axial pressure distribution (top right), and axial density distribution (bottom right). Also shown is a comparison with Fokker-planck code. 
Table III. Microstability Characteristics.

\begin{tabular}{|c|c|c|c|}
\hline$\cdot$ & $T M X$ & $T M X-U$ & Theory \\
\hline \multicolumn{4}{|l|}{ Microstability parameters: } \\
\hline$r_{p} / a_{i}$ & 5 & 4 & -- \\
\hline$L_{p} / 2 a_{j}$ & 10 & 25 & -- \\
\hline \multicolumn{4}{|l|}{ End-P]ug Fluctuation Characteristics: } \\
\hline Maximum amplitude (Volts at $2 r_{p}$ ) & 5 & $\uparrow$ & $?$ \\
\hline Mode & AIC & Loss cone & $\checkmark$ \\
\hline Frequency $\left(\omega / \omega_{\text {cio }}\right)$ & 0.85 & 1.9 & $\sqrt{ }$ \\
\hline Bandwidth $(\Delta w / \omega)$ & $<0.02$ & $<0.1$ & $?$ \\
\hline Wave length $\left(k_{1} a_{i 0}\right)$ & 0.3 & 7 & $\checkmark$ \\
\hline \multicolumn{4}{|l|}{ Phase velocity } \\
\hline (Diamagnetic direction) & Electron & Ion & $\checkmark$ \\
\hline Bursting & Yes & No & $?$ \\
\hline Propagation to central cel] & Yes & No & $\gamma$ \\
\hline
\end{tabular}


Compared to the TMX Alfuén-ion-cyclotron mode, the bandwidth for TMX-U is larger, the wavelength is smalier, and we do not observe bursting. A very important difference is that in $T M X-U$, the fluctuations do not propagate to the central cell nor do they appear to interact strongly with the central-cell ions. All these characteristics are in qualitative agreement with theory.

Further evidence of improved sloshing-ion microstability is seen in the energy spectra of central-cell ions escaping through the end plugs to the end walls, as shown in Fig. 11. This data compares TMX and TMX-U, each operating at $0.4-k V$ potential so that the loss-cone drive is comparable. In TMX, the 75-eV escaping-centra]-cell ions were heated to $800 \mathrm{eV}$ by the fluctuations as they passed through the p.ug. In TMX-U the rf heating is hardly measurable.

\section{SUMMARY}

The results of the sloshing-ion-mode experiments follow, highlighted as in Appendix A.

We generated a steady-state sloshing-ion tandem-mirror configuration.

1. Energy-confinement times up to $5 \mathrm{~ms}$ were measured. This was more than three times larger than we recorded in TMX. Central-cell betas were up to $10 \%$ and were up to 18 times the plug betas. No MHD limit was observed as the central-cell beam power was increased.

2. Plasma potentials up to $800 \mathrm{~V}$ were measurad.

3. No impurity problems were encountered.

4. Compared to TMX, improved operation was achieved, such as fewer shots were required for cleanup to establish full-duration shots. This is due to better vacuum conditions.

5. We demonstrated that low-density plasmas $\left(5 \times 10^{12} \mathrm{~cm}^{-3}\right)$, as required for thermal-barrier operation, can be sustained by neutral-beam injection.

- We demonstrated that the angular distribution of sloshing ions remains peaked, with hot-ion fractions exceeding 50\%. A comparison was made with the Fokker-planck code. 
- At equal potentials - negligible RF heating of central cell end loss ions

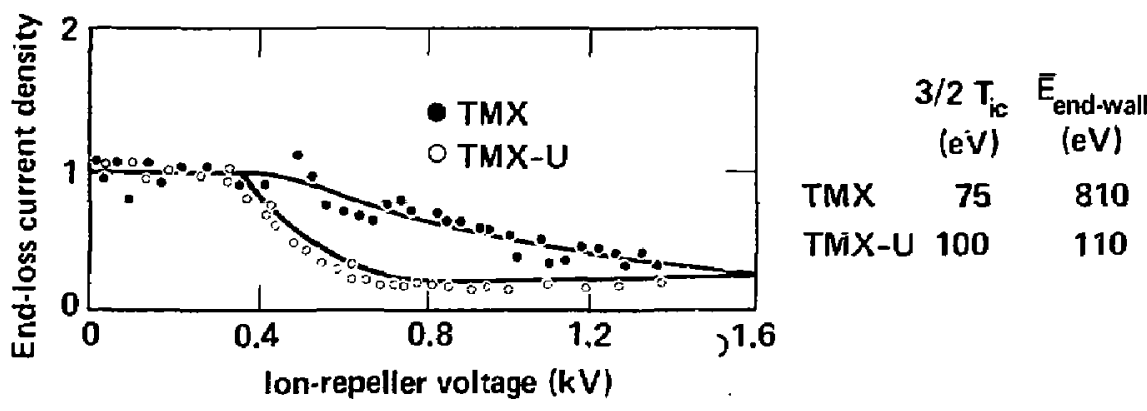

Fig. 11. Energy spectrum of central-cell ions escaping through the end plugs indicates less rf heating in JMX-U compared to TMX. 
- He observed no degradation in confinement because of microinstabilities and observed negligible heating of escaping central-celi ions by end-plug $r f$. For the first time in a beam-driven mirror machine, we did not observe fluctuations with the plug midplane ion-cyclotron frequency. Low-amplitude fluctuations observed have the characteristics predicted by theory for the sloshing-ion operating mode. - We also initiated ECRH experiments using one gyrotron. A key result was that we demonstrated startup without plasma guns. In other ECRH experiments, without neutral beams in the end ceil containing the gyrotron, we produced $2 \%$ hot-electron betas and obtained. $10 \%$ heating efficiency. We find these preliminary ECRH experiments encouraging as they are consistent with our theoretical hot-electron models for the thermal-barrier operating mode. Continuation of these experiments with four gyrotrons is the main objective of the october experimental run. 
APPENDIX A

\section{TMX-UPGRADE SL.OSHING ION EXPERIMENTAL RESULTS}

- Generated steady state tandem mirror configuration

$-5 \mathrm{~ms}$ energy confinement times ( 3 times longer than TMiX)

$-10 \%$ central cell betas $\left(\beta_{c} / \beta_{p}\right.$ up to 18$)$ no MHD limit observed

$-0.8 \mathrm{kV}$ potentials

- Low $1 \%$ impurity concentrations $(O, N, C)$

- Demonstrated sloshing ion end plug

- Angular distribution remains peaked

$-\mathrm{n}_{\mathrm{jHOT}} / \mathrm{n}_{\mathrm{e}}=0.5$ to 0.8

- Comparison with Fokker Planck code

- Observed no degradation in confinement due to microinstabilities

- Plasma parameters do not change with changing fluctuation amplitude

- No fluctuations with midplane ion cyclotron frequency

- AIC mode observed in TMX stabilized

- Low level fluctuations have characteristics predicted by theory

- Initiated $28 \mathrm{GHz}$ ECRH experiments

- ECRH startup without plasma guns

- $2 \%$ irot electron betas produced with $40 \mathrm{~kW}$

- $10 \%$ heating efficiency consistent with electron Fokker Planck code 


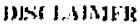

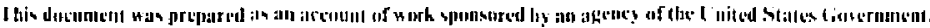

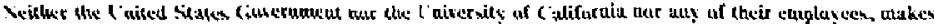
any warranty, axpress or implied, or astumes any legal liahitity or responsihility for the accuract. com-

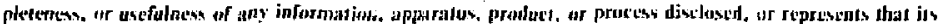

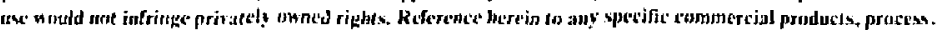

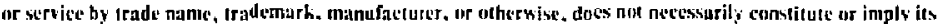

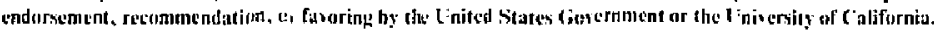

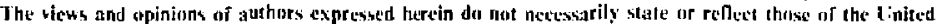
States couscroment thurear, and shall not he exed for advertising of product endorsement purposes.

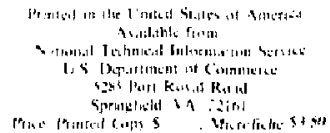

\begin{tabular}{|c|c|c|c|}
\hline Page Kange & $\begin{array}{l}\text { Dojnestie } \\
\text { Price }\end{array}$ & Page Rangt & $\begin{array}{l}\text { Domestic } \\
\text { Price }\end{array}$ \\
\hline $001-025$ & $\$ 5.00$ & $326-350$ & $=88.00$ \\
\hline $026-0,50$ & 6.00 & $351-375$ & 19.00 \\
\hline $051-075$ & 7.00 & $376-400$ & 20.00 \\
\hline $076 \cdot 100$ & 8.00 & $401-425$ & 21.00 \\
\hline $101-125$ & 9.90 & 426.450 & 22.00 \\
\hline $326-150$ & 10.00 & 451.475 & 23.00 \\
\hline 151.175 & 51,00 & $476-500$ & 24.00 \\
\hline $176-200$ & 12.00 & $50]-525$ & 35.00 \\
\hline $201-225$ & 13.00 & $526-550$ & 26.00 \\
\hline $226-250$ & 14.00 & $551-525$ & 27,00 \\
\hline $2.51-275$ & 15.00 & $526-550$ & 28.00 \\
\hline $276-300$ & 16.00 & $6011-u \mid 1$ & \\
\hline $301-325$ & 17.50 & & \\
\hline
\end{tabular}

${ }^{3}$ Add 2.00 for each additional 25 puge increntent from 601 pages up. 
Technical Information Deparment - Lawrence Livermore Laboratory University of California - Livermore, California 94550 